\title{
Nefropatía asociada a poliomavirus BK en trasplante renal
}

\author{
BK polyomavirus-associated nephropathy in kidney transplant
}

\author{
Pedro Méndez Chacón ${ }^{1, a, 2, b}$, Nancy Guzmán Cuba ${ }^{2, b}$, José Somocurcio Peralta ${ }^{1,2, c}$, \\ Armando Vidalón Fernández ${ }^{1, a, 2, b}$, Rafael Villacana ${ }^{3, d}$, Carla Méndez Chacón ${ }^{4, c}$ \\ ${ }^{1}$ Facultad de Medicina Humana, Universidad Nacional Mayor de San Marcos, Lima, Perú. \\ ${ }^{2}$ Hospital Nacional Edgardo Rebagliati Martins, EsSalud, Lima, Perú. \\ ${ }^{3}$ Cedars Sinai Medical Center, EE UU. \\ ${ }^{4}$ Hospital Nacional Guillermo Almenara Irigoyen, EsSalud, Lima, Perú. \\ ${ }^{a}$ Docente; ${ }^{b}$ Médico Asistente, Departamento de Nefrología; ${ }^{\circ}$ Médico Asistente, Departamento Patologia Clinica; \\ ${ }^{\mathrm{d}}$ Médico Adjunto, Unidad de Trasplante Renal.
}

\begin{abstract}
Resumen
El objetivo del presente reporte es dar a conocer los dos primeros casos de nefropatía del injerto asociada a poliomavirus BK (NPBK) ocurridos en el Hospital Edgardo Rebagliati Martins, EsSalud, Lima, Perú. El diagnóstico definitivo de la NPBK se corroboró mediante biopsia renal y estudio de microscopia electrónica. En el caso 1, el deterioro funcional renal no se controló a pesar de reducir la inmunosupresión y añadir drogas antivirales (leflunamide y ciprofloxacina), evolucionando a falla renal y su posterior acceso a retrasplante renal. En el caso 2, la alternativa terapéutica se basó en infusión de inmunoglobulina endovenosa asociada a reducción de la inmunosupresión, resultando en moderada mejora histológica y estabilización de la función renal. En ambos injertos renales hubo concomitantemente NPBK y lesiones histológicas compatibles con rechazo agudo celular pendientes de interpretar. En conclusión, la presencia del poliomavirus BK representa serio problema en el riñón trasplantado. La mejor conducta terapéutica se basa en el diagnóstico precoz y subsecuente reducción de la inmunosupresión. Es imprescindible disponer de metodología apropiada que posibilite un diagnóstico preciso. Se utiliza tamizaje de células señuelo o marcadoras (decoy cells) en orina, reacción de cadena polimerasa (PCR), biopsia renal o la microscopia electrónica como método valioso de ayuda en el diagnóstico etiológico viral. Se considera el retrasplante como opción ante la pérdida del injerto por NPBK (caso 1).
\end{abstract}

Palabras clave. Poliomavirus BK; Nefropatía; Trasplante de riñón; Inmunología.

\section{Abstract}

Two documented cases of BK polyomavirus-associated nephropathy (BKVN) seen at Hospital Edgardo Rebagliati Martins, EsSalud, Lima, Peru are reported. Final BKVN diagnosis was confirmed by renal biopsy and electron microscopy study. Case 1: Renal functional deterioration was not controlled despite reduction of immunosuppression and addition of antiviral drugs (leflunomide and ciprofloxacin), evolving to renal failure and subsequent kidney retransplantation. Case 2: The therapeutical management consisted in intravenous immunoglobulin infusion linked to reduction of immunosuppression; this resulted in modest histological improvement and stabilization of renal function. Both renal grafts concomitantly presented BKVN and histological lesions consistent with acute rejection, pending interpretation. In conclusion presence of BK polyomavirus is a serious problem for transplanted kidneys. The best treatment is based on early diagnosis and subsequent reduction of immunosuppression. It is essential to have an appropriate methodology for precise diagnosis. Early electron microscopy is a valuable method for viral etiologic diagnosis. Retransplantation is considered a treatment option when faced with possible BKVN-related graft loss (Case 1).

Keywords. BK polyomavirus; Nephropathy; Kidney transplantation; Immunology.

An Fac med. 2015;76(4):439-43 / http://dx.doi.org/10.15381/anales.v76i4.11415 


\section{INTRODUCCIÓN}

La nefropatía poliomavirus BK (NPBK) en el injerto renal constituye patología aún no documentada en nuestro hospital. Reportes americanos de centros de trasplante renal confirman sostenido incremento del porcentaje de su prevalencia $(5 \% \text { a } 10 \%)^{(1,2)}$. Emerge como una patología que puede ocasionar la pérdida del injerto en más de la mitad de los casos ${ }^{(3-8)}$.

La replicación viral resulta del complejo de interacciones entre paciente, injerto y virus, condicionadas por el mayor grado de inmunosupresión. Nuevas drogas inmunosupresoras crean un ambiente cada vez más propicio para la reactivación viral ${ }^{(9)}$.

Se reporta los dos primeros casos de poliomavirus detectados en nuestra casuística de 1440 trasplantes renales realizados en el Hospital Edgardo Rebagliati Martins (1969 a 2012).

La NPBK no tiene tratamiento satisfactorio antiviral definitivo. El diagnóstico temprano y la drástica reducción de la inmunosupresión representan únicas medidas probadas de manejo útil a realizar antes que ocurran cambios parenquimales irreversibles ${ }^{(10)}$. El retrasplante debe considerarse opción válida ante la pérdida del injerto por NPBK ${ }^{(11)}$, una vez negativizada la viremia.

\section{REPORTE DE CASOS}

\section{Caso 1}

Paciente varón de 27 años con historia de insuficiencia renal crónica terminal (IRCT) de causa no determinada. Recibió el primer trasplante renal de donante cadavérico de 23 años de edad (2 de diciembre de 2006), compartiendo tres identidades de antígenos leucocitarios humanos (HLA) y panel de anticuerpos reactivos $0 \%$. Isquemia total de 16 horas 30 minutos e isquemia caliente secundaria de 1 hora. No recibió terapia de inducción y la medicación de mantenimiento consistió en ciclos- porina, micofenolato mofetilo y prednisona. Su curso clínico postrasplante no tuvo complicaciones. Tuvo creatinina $1,2 \mathrm{mg} \%$, depuración de creatinina 68 $\mathrm{mL} / \mathrm{min}$, proteinuria $0,56 \mathrm{~g} / \mathrm{d}$ á; $\mathrm{PCR}$ para poliomavirus no fue estudiado por falta de logística hospitalaria. Se realizó biopsia renal en el décimo mes postrasplante por cursar con incremento de niveles de creatinina $(1,1$ a $2,6 \mathrm{mg} \%)$, dosaje sérico de tacrolimus $8 \mathrm{ng} / \mathrm{dL}$ y citología urinaria que evidenció células en señuelo (decoy cells) positivo (figura 2). La histología reveló nefritis intersticial de poliomavirus BK asociada a signos sugestivos de rechazo agudo celular grado I-A (estudios $\mathrm{C}_{4} \mathrm{~d}$ y DSA negativos). Al no disponer de estudios moleculares e inmunohistoquímica en tejido renal, se recurrió a la microscopia electrónica, descubriéndose células tubulares conteniendo inclusiones nucleares virales compatibles con poliomavirus subtipo BK. Luego de la evaluación histológica, se decidió reducción de la dosis de inmunosupresión, para luego instaurar terapia con leflunomida 20 $\mathrm{mg} /$ día y ciprofloxacino 500 mg/día. La biopsia renal de control reveló atrofia tubular y fibrosis intersticial severa. La diálisis se inició seis meses después de ocurrido el diagnóstico, procediéndose luego a la injertectomía.

Cinco años más tarde el paciente recibió segundo trasplante renal de dador cadavérico de 38 años de edad, compartiendo solo una identidad de HLA y estudios panel de anticuerpos reactivos (PRA) anti clase HLA-I 35\% y anti clase HLA-II 46\%. La seroconversión para virus de hepatitis $\mathrm{C}$ fue positiva. Recibió terapia inductiva con basiliximab y mantenimiento a base de ciclosporina, micofenolato mofetilo y prednisona. Seis meses después del trasplante, la función del injerto continuó estable con creatinina $1,1 \mathrm{mg} \%$ y estudios de citología urinaria decoy cells negativos.

\section{Caso 2.}

Paciente de 50 años con IRCT de causa no determinada y terapia de hemodiálisis durante dos años. Recibió trasplante renal de donante cadavérico de 15 años de edad. Compartieron 4 identidades de HLA y PRA fue $0 \%$. No hubo complicaciones en el postrasplante inmediato. La terapia inductiva fue con basiliximab y de mantenimiento con tacrolimus, micofenolato mofetilo y prednisona. Tuvo niveles de creatinina $1,3 \mathrm{mg} \%$ al momento del alta hospitalaria. Sometido a biopsia renal al año postrasplante por incremento de niveles de creatinina $(1,3$ a $2,6 \mathrm{mg} \%$ ) y citología urinaria decoy cells positivo, el estudio histológico reveló nefritis intersticial a poliomavirus BK asociado a sugestivos cambios de rechazo agudo celular IA (ausencia de capilaritis peritubular y DSA negativo). El estudio de PCR BK sérico identificó 22000 copias por $\mathrm{mL}$. Se decidió reducir la inmunosupresión. La biopsia renal de control reveló disminución de las lesiones histológicas de la NPBK (de estadio C a estadio B). Se añadió inmunoglobulina endovenosa 2 $\mathrm{g} / \mathrm{kg}$ (dosis única repartida en dos días), estabilizando la función renal paulatinamente.

\section{DISCUSIÓN}

La NPBK deviene en problema clínico recientemente detectado en nuestra actividad trasplantadora. Reportamos los dos primeros casos diagnosticados en 1400 trasplantes renales realizados en nuestro centro (1969 a 2012). Constituye patología infrecuente en nuestro medio o tal vez no diagnosticada ${ }^{(12)}$, probadamente relacionada a determinados esquemas que incluyen drogas inmunosupresoras recientes y/o sobredosis de las mismas ${ }^{(10,13-16)}$. Los regímenes actuales de inmunosupresión son más potentes e incrementan el riesgo de infecciones virales oportunistas presentes mayormente dentro del primer año ${ }^{(17)}$. Ambos pacientes fueron admitidos a hospitalización por aumento inexplicable de la creatinina sérica ${ }^{(18)}$, sin signos ni síntomas específicos de infección o rechazo. Las biopsias del injerto renal mostraron lesiones típicas de NPBK ${ }^{(19,20)}$ (figura 1). El diseño histológico de probable rechazo agudo celular sobreagregado fue de difícil in- 


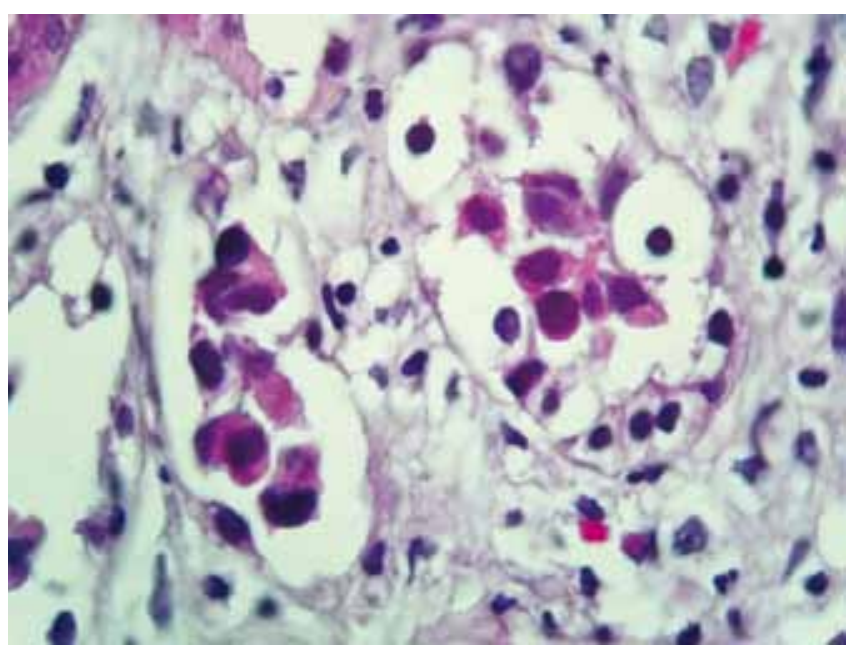

Figura 1. Nefropatía por poliomavirus tipo BK. Hallazgos histológicos en la biopsia de riñón trasplantado. Células epiteliales tubulares presentan cuerpos de inclusión viral intranuclear y severa alteración de la arquitectura tubular. Infiltrado inflamatorio mononuclear y tubulitis donde está la inclusión; este hallazgo sugiere que la tubulitis no es debida a rechazo.

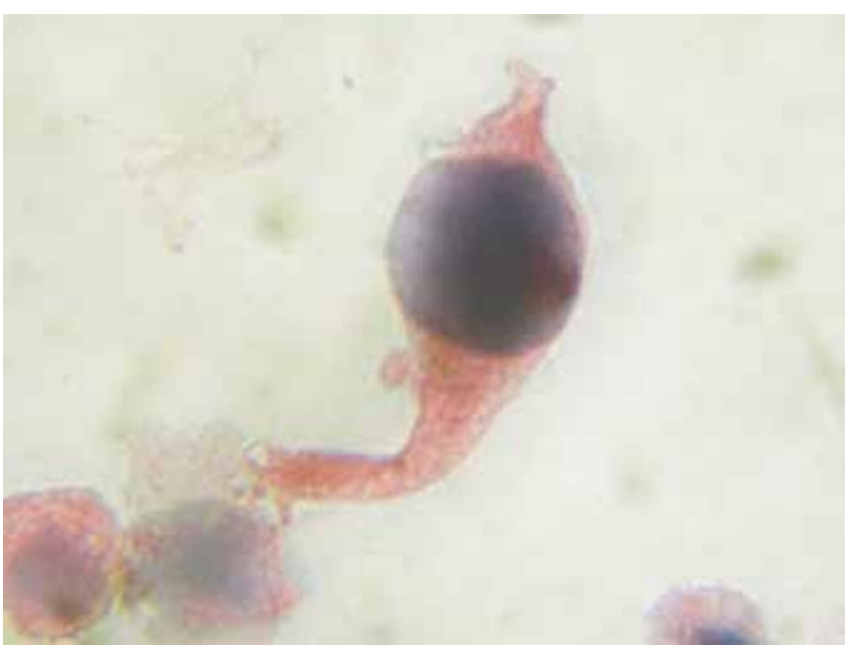

Figura 2. Citología urinaria. Decoy cells con aspecto 'en cometa' debido a alteraciones en el citoplasma. Observe que hay cuerpos de inclusión intranuclear. terpretación; el estudio inmunohistoquimico hubiese sido de gran ayuda ${ }^{(21)}$. La necrosis y denudación de la membrana basal tubular inicial severa asociada a atrofia tubular y fibrosis intersticial encontradas en las biopsias posteriores del injerto, fueron indicativas de evolución tórpida por diagnóstico tardío ${ }^{(22)}$, especialmente en el caso 1.

El monitoreo del virus $\mathrm{BK}$ urinario (decoy cells), cuya sensibilidad puede llegar hasta a $100 \%{ }^{(3,12,15)}$ (figura 2), es lo recomendable, antes que ocurra daño histológico renal ${ }^{(1,2,19,23)}$. Sin embargo, las decoy cells urinarias también han sido descritas en cuadros de adenovirus, citomegalovirus (CMV) y células malignas ${ }^{(24)}$. Definitivamente, la aplicación de técnicas moleculares, reacción en cadena de la polimerasa (PCR), virus BK sérico/orina constituyen el instrumento fundamental de diagnóstico etiológico ${ }^{(3,20)}$.

Los expertos recomiendan la biopsia renal cuando la carga viral de $\mathrm{BK}$ sérica es permanentemente superior a 10000 copias/mL ${ }^{(3)}$, nivel de corte de carga viral, también recomendable para dar inicio a la reducción empírica de la inmunosupresión ${ }^{(3)}$. Aún no disponemos del uso regular de estas valiosas técnicas moleculares que permitan manejo anticipado de la infección de virus BK ${ }^{(26,35)}$. Cuando tuvimos el primer caso de sospecha de NPBK, recurrimos a la microscopia electrónica (ME) para identificar el virus al no disponer de reactivos para estudio inmunohistoquimico en la histología renal ${ }^{(2,24)}$ ni técnicas moleculares. El estudio de la $\mathrm{ME}$ nos permitió corroborar la presencia de genomas de virus BK (partículas típicas electrodensas de aspecto paracristalino tamaño de 40 a $50 \mathrm{~nm}$ ) (figura 3). La simultánea ocurrencia de NPBK y aparente imagen histológica de rechazo agudo en ambos casos constituyó un serio problema clínico de interpretación en la estrategia del manejo, es decir, las imágenes simultáneas pueden corresponder únicamente al virus ${ }^{(2,15)}$, optándose entonces por el pronto retiro de las drogas inmunosupresoras a fin de restaurar espontáneamente la inmunidad antiviral, elemento clave del tratamiento ${ }^{(3,14)}$. El uso de drogas antivirales constituye actualmente un problema controversial en sus resultados, optando nosotros por añadir leflunonamida ${ }^{(18,25-27)}$ y ciprofloxacina ${ }^{(18,34)}$ para el caso 1 y administración de inmunoglobulina endovenosa para el caso $2^{(28,29)}$, drogas antivirales todas ellas con ca- pacidad de inhibir la replicación viral $\mathrm{BK}$ in vitro ${ }^{(14)}$. El objetivo primario de la terapia es disminuir la replicación viral BK (VBK) sérico, que tiene que ver con la restauración de la inmunidad y su repercusión en la evolución de la histología renal y preservación de la función. Si no se tiene el apoyo del monitoreo de la carga VBK, los resultados serán infructuosos. En el caso 1, el deterioro de la función renal lamentablemente evolucionó a la pérdida del injerto, no obstante la aplicación de todas las estrategias recomendadas, aunque tardíamente ${ }^{(8)}$. Las pérdidas del injerto $(10-80 \%)$ son menores en aquellos centros de trasplante donde se realiza vigilancia molecular periódica de la infección de virus BK con instauración del manejo clínico terapéutico precoz. Ante la falta de tratamiento específico para la NPBK, el diagnóstico precoz y reducción o retiro de la inmunosupresión constituyen la conducta más oportuna. Las lesiones tisulares renales en las fases iníciales son reversibles ${ }^{(30)}$. Ocurrida la pérdida del primer injerto por virus $\mathrm{BK}$, el retrasplante representó una opción válida ${ }^{(21)}$. Este ha sido exitosamente realizado en aproximadamente 80 a $90 \%$ de los casos reportados ${ }^{(22)}$. Nuestro paciente retrasplan- 
tado con éxito usó las mismas drogas inmunosupresoras que para el primer injerto. El retrasplante no es una contraindicación. Minimizar la probabilidad de recurrencia de la replicación del VBK es una condición que debemos tener siempre presente. La recurrencia está reportada en un $15 \%{ }^{(2,21,31)} \mathrm{com}$ parando con $5 \%$ en el trasplante renal primario ${ }^{(21)}$. Se recomienda ausencia de replicación del VBK para su aceptación al retrasplante ${ }^{(14)}$. La remoción quirúrgica del primer riñón trasplantado no es requerimiento a menos que en un retrasplante anticipado aquel esté replicando. La remoción quirúrgica del injerto ha sido reportada en aproximadamente la mitad de todos los casos, sin evidencia de protección contra la recurrencia de la replicación de VBK. El caso 1 se sensibilizó inmunológicamente con el primer trasplante y el uso de timoglobulina como terapia inductiva para el retrasplante fue diferido por la seroconversión del virus de hepatitis C. Después de un seguimiento del postrasplante a los 6 meses, el injerto funciona bien y cursa con citología urinaria normal.

En el caso 2, optamos por el uso de la inmunoglobulina, también utilizada en el tratamiento de complicaciones in- fecciosas virales asociadas al trasplante, como CMV, enfermedad de virus Epstein Barr (EBV) / desorden linfoproliferativo postrasplante (PTLD) e infección parvovirus B19 ${ }^{(32,33)}$. Se describe que la inmunoglobulina posee acción de anticuerpo antipoliomavirus ${ }^{(33)}$. La racionalidad del uso de la inmunoglobulina en el manejo de NPBK está también basada en la potencial transferencia de su inmunidad protectora ${ }^{(32)}$. La eficacia de la inmunoglobulina es difícil de establecer debido a que es típicamente utilizada en conjunción con otras formas de tratamiento. Se reporta estabilización de la función renal, reducción de la viremia y resolución histológica de la NVBK luego de suspender la inmunosupresión y uso de inmunoglobulina (26). En nuestro paciente, la función renal se estabilizó temporalmente con recuperación parcial histológica de la NVBK.

En conclusión, el retiro de la inmunosupresión constituye la primera línea de intervención para conseguir la remisión virémica e histológica por el VBK ${ }^{(26,32)}$. Las predicciones de una buena respuesta terapéutica a los antivirales no está bien documentada (26). La efectividad de la inmunoglobulina endovenosa ofrece una alternativa de

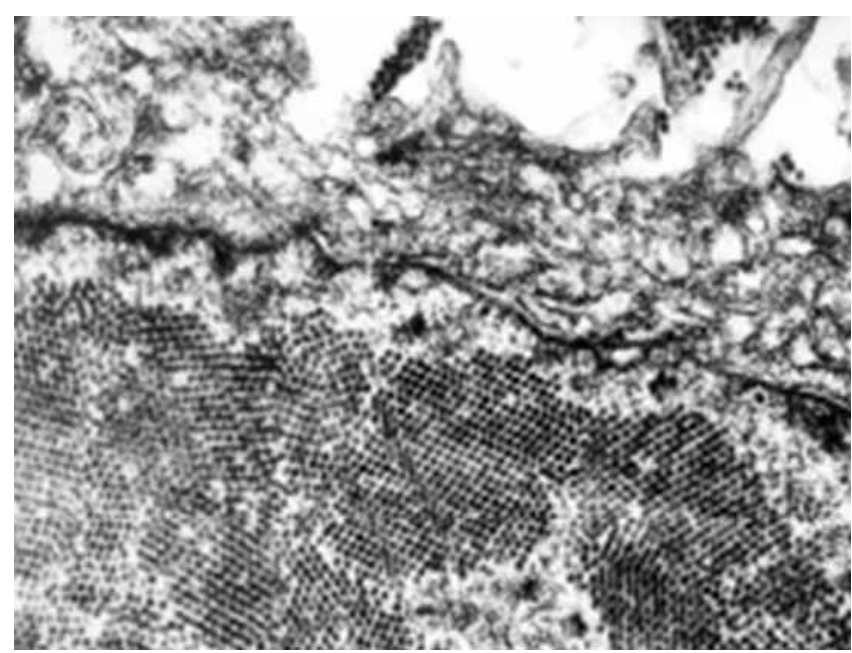

Figura 3. Nefropatía por poliomavirus tipo BK. Microscopia electrónica de la biopsia renal mostrando en el núcleo de una célula epitelial tubular agregados de partículas virales configurando estructuras paracristalinas. tratamiento que necesita ser explorada. La inmunosupresión intensa y el diagnóstico tardío predisponen a la tórpida evolución de la NPBK. En etapas tempranas del postrasplante es importante el monitoreo con los métodos de diagnóstico apropiados. Los pacientes con NPBK tienen reducida sobrevida del injerto a largo plazo. Queda por definirse la validez de la imagen adicional sugestiva de rechazo agudo como propia de la patología viral, esto de gran importancia en el manejo terapéutico.

\section{REFERENCIAS BIBLIOGRÁFICAS}

1. Jay AF. BK nephropathy: What is the role of antiviral therapy? Am J Transplantation. 2003;3(2):99-100. doi: 10.1034/j.1600-6143.2002.020106.x.

2. Hirsch HH, Brennan DC, Drachenberg CB, Ginevri F, Gordon J, Limaye, AP; Mihatsch MJ, et al. Polyomavirus-associated nephropathy in renal transplantation: interdisciplinary analyses and recommendations. Transplantation. 2005;79(10):1277-86.

3. Viscount HB, Eid AJ, Espy MJ, Griffin MD, Thomsen KM, Harmsen WS, et al. Polyomavirus polymerase chain reaction as a surrogate marker of polyomavirus-associated nephropathy. Transplantation. 2007;84(3):340-5. doi:10.1097/01. tp.0000275205.41078.51.

4. Ramos E, Hirsch HH. Polyomavirus-associated nephropathy: updates on a persisting challenge. Transplant Infect Dis. 2006;8(2):59-61. doi: 10.1111/j.1399-3062.2006.00164.x.

5. Gai M, Lanfranco G, Segoloni GP. "Decoy cells" in urine. Transplantation Proc. 2005;37(10):430910. doi: http://dx.doi.org/10.1016/j.transproceed.2005.11.045.

6. Josephson MA, Gillen D, Javaid B, Kadamb $\mathrm{P}$, Meeha, S, Foster $\mathrm{P}$, et al. Treatment of renal allograft polyoma BK virus infection with leflunomide. Transplantation. 2006;81(5):704-10. doi: 10.1097/01.tp.0000181149.76113.50.

7. Haysom L, Rosenberg AR, Kainer G, Waliuzzaman ZM, Roberts J, Rawlinson WD, et al. BK viral infection in an Australian pediatric renal transplant population. Pediatric Transplantation. 2004;8(5):480-4. doi: 10.1111/j.1399-3046.2004.00154.x.

8. Josephson MA, Williams JW, Chandraker A, Randhawa PS. Polyomavirus-associated nephropathy: update on antiviral estrategies .Transplant Infect Dis. 2006;8(2):95-101. doi: 10.1111/j.13993062.2006.00150.x

9. Hirsch $\mathrm{HH}$, Randhawa P, the AST Infectious Diseases Community of Practice. BK virus in solid organ transplant recipients. Am J Transplantation. 2009;9(s4):S136-S146. doi: 10.1111/j.16006143.2009.02904.x.

10. Hirsch HH, Ramos E. Retransplantation after polyomavirus-associated nephropathy: just do it? Am J Transplantation. 2006;6(1):7-9.doi:10.1111/j.16006143.2005.01171.x.

11. Poduval RD, Meehan SM, Steve WE, Richard TJ, Haas M, Cronin DC, et al. Successful retransplantation after renal allograft loss to polyoma virus interstitial nephritis. Transplantation. 2002;73(7):1166-9. 
12. Nickeleit $V$, Hirsch $H H$, Binet IF, Gudat F, Prince $\mathrm{O}$, Dalquen $\mathrm{P}$, et al. Polyomavirus infection of renal allograft recipients: from latent infection to manifest disease. JASN .1999;10(5):1080-9.

13. Doucette KE, Pang XL, Jackson K, Burton I, Carbonneau M, Cockfield S, et al. Prospective monitoring of BK polyomavirus infection early posttransplantation in nonrenal solid organ transplant recipients. Transplantation. 2008;85(12):1733-6. doi: $10.1097 /$ TP.0b013e3181722ead.

14. Trofe J, Hirsch HH, Ramos E. Polyomavirusassociated nephropathy: update of clinical management in kidney transplant patients. Transplant Infect Dis. 2006;8(2):76-85.

15. Bohl DL, Brennan DC. BK virus nephropathy and Kidney transplantation. CJASN. 2007;2(1):S36S46.

16. Thomas A, Dropulic LK, Rahman MH, Geetha D. Ureteral Stents: A novel risk factor for polyomavirus nephropathy. Transplantation. 2007;84 (3):433436. doi: 10.1097/01.tp.0000269616.21698.10.

17. Buehrig CK, Lager DJ, Stegall MD, Kreps MA, Kremers WK, Gloor JM, et al. Influence of surveillance renal allograft biopsy on diagnosis and prognosis of polyoma virus associated nephropathy. Kidney Internat. 2003;64:665-73. doi:10.1046/j.15231755.2003.00103.x.

18. Randhawa P, Brennan DC. BK virus infection in transplant recipients: an overview and update. Am J Transplantation. 2006;6(9):2000-5.doi: 10.1111/j.1600-6143.2006.01403.x.

19. Womer KL, Guerra G, Dibadj K, Huang Y, Kazory A, Kaplan B, et al. Immunosuppression reduction for BK virus nephropathy: A case for caution. Transplant Infect Dis. 2007;9(3):244-8. doi: 10.1111/j.1399-3062.2007.00204.x.

20. Ramos E, Drachenberg CB, Papadimitriou JC, Hamze O, Fink JC, Klassen DK, et al. Clinical course of polyoma virus nephropathy in 67 renal transplant patients. JASN. 2002;13(8):2145-51.

21. Hirsch HH, Brennan DC, Drachenberg CB, Ginevri F, Gordon J, Limaye, AP, et al. Polyomavirusassociated nephropathy in renal transplantation: interdisciplinary analyses and recommendations. Transplantation. 2005;79(10):1277-86.
22. Dadhania D, Snopkowski C, Ding R, Muthukumar T, Chang C, Aull M, et al. Epidemiology of BK virus in renal allograft recipients: identification of steroid maintenance therapy and rabbit anti-human thymocyte globulin induction as independent risk factors for BKV replication. Transplantation. 2008;86(4):521-8. doi: 10.1097/ TP.0b013e31817c6447.

23. Mathur VS, Olson JL, Darragh TM, Yen TSB Polyomavirus-induced interstitial nephritis in two renal transplant recipients: Case reports and review of the literature. AJKD. 1997;29(5):854-8. doi: 10.1016/S0272-6386(97)90130-X.

24. Bracamonte E, Leca N, Smith KD, Nicosia RF, Nickeleit V, Kendrick E, et al. Tubular basement membrane immune deposits in association with BK polyomavirus nephropathy. Am J Transplantation. 2007;7(6):1552-60. doi:10.1111/j.1600-6143.2007. 01794.x.

25. Chong AS, Zeng $H$, Knight DA, Shen J, Meister GT, Williams JW, et al. Concurrent antiviral and immunosuppressive activities of leflunomide in vivo. Am J Transplantation. 2006;6(1):69-75. doi: 10.1111/j.1600-6143.2005.01152.x.

26. Teschner S, Geyer M, Wilpert J, Schwertfeger E , Schenk T, Walz G, et al. Remission of polyomavirus-induced graft nephropathy treated with lowdose leflunomide. Nephrol Dialysis Transplantation. 2006;21(7):2039-40.

27. Maggiore U, Medici MC, Vaglio A, Buzio C. Increased viral load after intravenous immunoglobulin therapy for BK virus-associated nephropathy. Transplant Infect Dis. 2010;12(5):470-2. doi: 10.1111/j.1399-3062.2010.00512.x.

28. Anyaegbu El, Almond PS, Milligan T, Allen WR Gharaybeh S, Al-Akash SI. Intravenous immunoglobulin therapy in the treatment of BK viremia and nephropathy in pediatric renal transplant recipients. Pediatr Transplant. 2012;16(1):E19-24. doi: 10.1111/j.1399-3046.2010.01384.x.

29. Sola E, García I, Burgos D, Cabello M, Alférez MJ, López J, et al. Nefropatía por poliomavirus tipo BK en el trasplante renal. Nefrologia. 2001;21(6):548-55.
30. Womer KL, Meier-Kriesche HU, Patton PR, Dibadj K, Bucci CM, Foley D, et al. Preemptive retransplantation for BK virus nephropathy: successful outcome despite active viremia. Am J Transplantation. 2006;6(1):209-13. doi: 10.1111/j.16006143.2005.01137.x.

31. Smith JM, Jordan SC. Intravenous immunoglobulin as treatment for BK virus: nephropathy. Pediatr Transplant. 2009;13(1):11-3. doi: 10.1111/j.13993046.2008.01105.x.

32. Sener A, House AA, Jevnikar AM, Boudville N, McAlister VC, Muirhead N, et al. Intravenous immunoglobulin as a treatment for BK virus associated nephropathy: one-year follow-up of renal allograft recipients. Transplantation. 2006;81(1):117-20.

33. Bonvoisin C, Weekers L, Xhignesse P, Grosch S, Milicevic M, Krzesinski JM. Polyomavirus in renal transplantation: a hot problem. Transplantation. 2008;85(7S):S42-S48. doi: 10.1097/ TP.0b013e318169c794.

34. Hardinger KL, Koch MJ, Bohl DJ, Storch GA, Brennan DC. BK-virus and the impact of pre-emptive immunosuppression reduction: 5-year results. Am J Transplantation. 2010;10(2):407-15. doi : 10.1111/j.1600-6143.2009.02952.x.

Artículo recibido el 16 de febrero de 2015 y aceptado para publicación el 27 de agosto de 2015.

Fuente de financiamiento: Ninguno.

Conflictos de interés: Ninguno.

Correspondencia:

Dr. Pedro Méndez Chacón

Dirección: Av. Caminos de Inca No 2439, Santiago de Surco, Lima, Perú

Correo electrónico:pjmendez2010@hotmail.com 